Acta Crystallographica Section E

Structure Reports

Online

ISSN 1600-5368

\section{Bis(2-amino-4-phenyl-1,3-thiazol-3-ium) tetrachloridopalladate(II)}

\section{Reyna Reyes-Martínez, ${ }^{a}$ Rubén M. Carballo, ${ }^{a}$ Gonzalo J. Mena-Rejón, ${ }^{a}$ Simón Hernández-Ortegab and David Cáceres-Castillo ${ }^{\mathrm{a} *}$}

a Facultad de Química, Universidad Autónoma de Yucatán, Calle 41 No. 421, Col Industrial, CP97150, Mérida, Yucatán, Mexico, and ${ }^{\mathbf{b}}$ Instituto de Química, Universidad Nacional Autónoma de México, Circuito exterior, Ciudad Universitaria, México, DF, 04510, Mexico

Correspondence e-mail: david.caceres@uady.mx

Received 18 June 2014; accepted 1 July 2014

Edited by T. J. Prior, University of Hull, England

Key indicators: single-crystal X-ray study; $T=298 \mathrm{~K}$; mean $\sigma(\mathrm{C}-\mathrm{C})=0.003 \AA$; $R$ factor $=0.020 ; w R$ factor $=0.051 ;$ data-to-parameter ratio $=14.4$.

The title compound, $\left(\mathrm{C}_{9} \mathrm{H}_{9} \mathrm{~N}_{2} \mathrm{~S}\right)_{2}\left[\mathrm{PdCl}_{4}\right]$, consists of two monoprotonated 2-amino-4-phenyl-1,3-thiazole molecules and one tetrachloridopalladate anion. The organic molecules exhibit a dihedral angle between the main rings planes of $31.82(9)^{\circ}$. In the anion, the $\mathrm{Pd}^{\mathrm{II}}$ atom is located on a crystallographic centre of symmetry with a square-planar geometry. In the crystal, the anions and cations are connected through bifurcated $\mathrm{N}-\mathrm{H} \cdots \mathrm{Cl}$ hydrogen bonds, and these interactions lead to hydrogen-bonded tapes of cations and anions along [100].

\section{Related literature}

For the potential biological activity of compounds containing thiazole rings, see: Annadurai et al. (2012); Alam et al. (2011). For the synthesis of thiazole compounds, see: Cáceres-Castillo et al. (2012). For similar structures with protonated molecules, see: Form et al. (1974); Jin et al. (2011, 2013). For the crystal structure of non-protonated thiazole, see: Au-Alvarez et al. (1999).

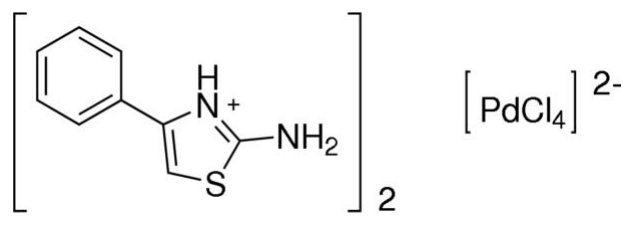

\section{Experimental}

Crystal data

$\left(\mathrm{C}_{9} \mathrm{H}_{9} \mathrm{~N}_{2} \mathrm{~S}\right)_{2}\left[\mathrm{PdCl}_{4}\right]$

$M_{r}=602.68$

Triclinic, $P \overline{1}$

$$
\begin{aligned}
& \alpha=66.258(1)^{\circ} \\
& \beta=73.778(1)^{\circ} \\
& \gamma=84.468(1)^{\circ} \\
& V=561.04(3) \AA^{3} \\
& Z=1
\end{aligned}
$$

Data collection

Bruker APEXII CCD area-detector diffractometer

Absorption correction: analytical (SADABS; Bruker, 2012)

$T_{\min }=0.658, T_{\max }=0.842$

\section{Refinement}

$R\left[F^{2}>2 \sigma\left(F^{2}\right)\right]=0.020$

$w R\left(F^{2}\right)=0.051$

$S=1.11$

2060 reflections

143 parameters

3 restraints

Table 1

Hydrogen-bond geometry $\left(\AA,^{\circ}\right)$.

\begin{tabular}{lllll}
\hline$D-\mathrm{H} \cdots A$ & $D-\mathrm{H}$ & $\mathrm{H} \cdots A$ & $D \cdots A$ & $D-\mathrm{H} \cdots A$ \\
\hline $\mathrm{N} 2-\mathrm{H} 2 B \cdots \mathrm{Cl} 2$ & $0.89(1)$ & $2.41(2)$ & $3.237(2)$ & $155(2)$ \\
$\mathrm{N} 2-\mathrm{H} 2 A \cdots \mathrm{Cl} 2^{\mathrm{i}}$ & $0.89(1)$ & $2.78(2)$ & $3.3572(19)$ & $123(2)$ \\
$\mathrm{N} 2-\mathrm{H} 2 A \cdots \mathrm{Cl} 1^{\mathrm{ii}}$ & $0.89(1)$ & $2.44(1)$ & $3.291(2)$ & $159(2)$ \\
$\mathrm{N} 1-\mathrm{H} 1 \cdots \mathrm{Cl} 2$ & $0.88(1)$ & $2.79(2)$ & $3.4028(17)$ & $129(2)$ \\
$\mathrm{N} 1-\mathrm{H} 1 \cdots \mathrm{Cl} 1$ & $0.88(1)$ & $2.49(2)$ & $3.2593(17)$ & $147(2)$ \\
\hline
\end{tabular}

Symmetry codes: (i) $-x+2,-y,-z+2$; (ii) $x+1, y, z$.

Data collection: APEX2 (Bruker, 2012); cell refinement: SAINT (Bruker, 2012); data reduction: SAINT; program(s) used to solve structure: SHELXTL (Sheldrick, 2008); program(s) used to refine structure: SHELXL97 (Sheldrick, 2008); molecular graphics: ORTEP-3 for Windows (Farrugia, 2012) and DIAMOND (Brandenburg, 2006); software used to prepare material for publication: SHELXTL and PLATON (Spek, 2009).

The authors from the Universidad Autónoma de Yucatán are grateful to Dr Leovigildo Quijano for assistance with the X-ray analysis.

Supporting information for this paper is available from the IUCr electronic archives (Reference: PJ2013).

\section{References}

Alam, M. S., Liu, L., Lee, Y.-E. \& Lee, D.-U. (2011). Chem. Pharm. Bull. 5, 568-573.

Annadurai, S., Martinez, R., Canney, D. J., Eidem, T., Dunman, P. M. \& AbouGharbia, M. (2012). Bioorg. Med. Chem. Lett. 22, 7719-7725.

Au-Alvarez, O., Peterson, R. C., Acosta Crespo, A., Rodríguez Esteva, Y., Marquez Alvarez, H., Plutín Stiven, A. M. \& Pomés Hernández, R. (1999). Acta Cryst. C55, 821-823.

Brandenburg, K. (2006). DIAMOND. Crystal Impact GbR, Bonn, Germany. Bruker (2012). APEX2, SAINT and $S A D A B S$. Bruker AXS Inc., Madison, Wisconsin, USA.

Cáceres-Castillo, D., Carballo, R. M., Tzec-Interián, J. A. \& Mena-Rejón, G. J. (2012). Tetrahedron Lett. 53, 3934-3936.

Farrugia, L. J. (2012). J. Appl. Cryst. 45, 849-854.

Form, G. R., Raper, E. S. \& Downie, T. C. (1974). Acta Cryst. B30, 342-348. Jin, S., Wang, D. \& Xu, Y. (2011). J. Chem. Crystallogr. 41, 1876-1883. Jin, S., Zhu, Q., Wei, S. \& Wang, D. (2013). J. Mol. Struct. 1049, 132-148. Sheldrick, G. M. (2008). Acta Cryst. A64, 112-122. Spek, A. L. (2009). Acta Cryst. D65, 148-155. 


\section{supporting information}

Acta Cryst. (2014). E70, m295 [doi:10.1107/S1600536814015360]

\section{Bis(2-amino-4-phenyl-1,3-thiazol-3-ium) tetrachloridopalladate(II) \\ Reyna Reyes-Martínez, Rubén M. Carballo, Gonzalo J. Mena-Rejón, Simón Hernández-Ortega and David Cáceres-Castillo}

\section{S1. Introduction}

The thiazole ring system is an important structural motif found in numerous molecules with potential biological activities, for instance; as antiinfective agents (Annadurai et al., 2012; Alam et al., 2011). On the other hand, in recent years there has been a growing interest in organic derivatives of transition metals in order to modify the biological properties of these organic compounds. Thus, in this opportunity we would like to report the crystal structure of bis-(2-amino-4-phenyl-1,3thiazolium) tetrachloropalladate (II).

\section{S2. Experimental}

\section{S2.1. Synthesis and crystallization}

The compound 2-amino-4-phenyl-1,3-thiazole was synthesized as reported by our group (Cáceres-Castillo et al., 2012). The $\mathrm{PdCl}_{2}(25 \mathrm{mg}, 0.14 \mathrm{mmol})$ was dissolved in $1 \mathrm{~mL}$ of concentrated $\mathrm{HCl}$ and then diluted with $5 \mathrm{~mL}$ of methanol. To the resulting mixture a methanol $(5 \mathrm{~mL})$ solution of 2-amino-4-phenyl-1,3-thiazole $(50 \mathrm{mg}, 0.28 \mathrm{mmol})$ was added. The reaction mixture was stirred for four hours at room temperature, after which time the resulting solution was allowed to slowly evaporate to produce brown X-ray diffraction quality crystals after few days.

\section{S2.2. Refinement}

Crystal data, data collection and structure refinement details are summarized in Table 1.

All $\mathrm{H}$ atoms were included in calculated positions $(\mathrm{C}-\mathrm{H}=0.93 \AA)$, and refined using a riding model with $\mathrm{Uiso}(\mathrm{H})=$ 1.2 Ueq of the carrier atom. $\mathrm{H}$ atoms on $\mathrm{N}$ were located in a Fourier map and refined isotropically with $\mathrm{Uiso}(\mathrm{H})=1.2 \times$ $\mathrm{Ueq}(\mathrm{N})$.

13 badly-fitted reflections were omitted from the final refinement.

\section{S3. Results and discussion}

The title compound, $\left[\mathrm{C}_{9} \mathrm{H}_{9} \mathrm{~N}_{2} \mathrm{~S}\right]_{2}\left[\mathrm{PdCl}_{4}\right]$, is centrosymmetric and consists of two monoprotonated 2-amino-4-phenyl-1,3thiazole molecules and one tetrachloropalladate anion. This compound, crystallized in the triclinic P-1 space group. The asymmetric unit is composed of one monoprotaned 2-amino-4-phenyl-1,3-thiazole and half of the tetrachloropalladate anion, the other half is generated by application of an inversion centre. The dihedral angle between the planes of the phenyl and thiazole rings in the cation is of $31.82(9)^{\circ}$.This value is larger than those reported in other compounds containing the 2-amino-4-phenyl-1,3-thiazole molecule, protonated (Form et al., 1974; Jin et al., 2013; Jin et al., 2011) or in the free molecule (Au-Alvarez et al., 1999). The angle $\mathrm{C} 2-\mathrm{N} 1-\mathrm{C} 5\left(115.25(17)^{\circ}\right)$ is similar in value other salts reported and is longer than that reported for the neutral compound $\left(110.5^{\circ}\right)$. 
The palladium atom of the anion is in a special position $(0.5,0,1)$, Wyckoff site $1 d$, and exhibits a square-planar geometry with $\mathrm{Pd}-\mathrm{Cl}$ distances of 2.3031 (5) and 2.3061 (6) $\AA$. The cation and the anion are linked by a bifurcate hydrogen bond between the chloride atoms and the hydrogen of the thiazole ring (Figure 1). The $\mathrm{NH}$ and $\mathrm{NH}_{2}$ groups exhibit $\mathrm{N}-\mathrm{H} \cdots \mathrm{Cl}$ hydrogen bonds with the chloride atoms generating a linear arrangement in the orientation [100] (Figure 2).

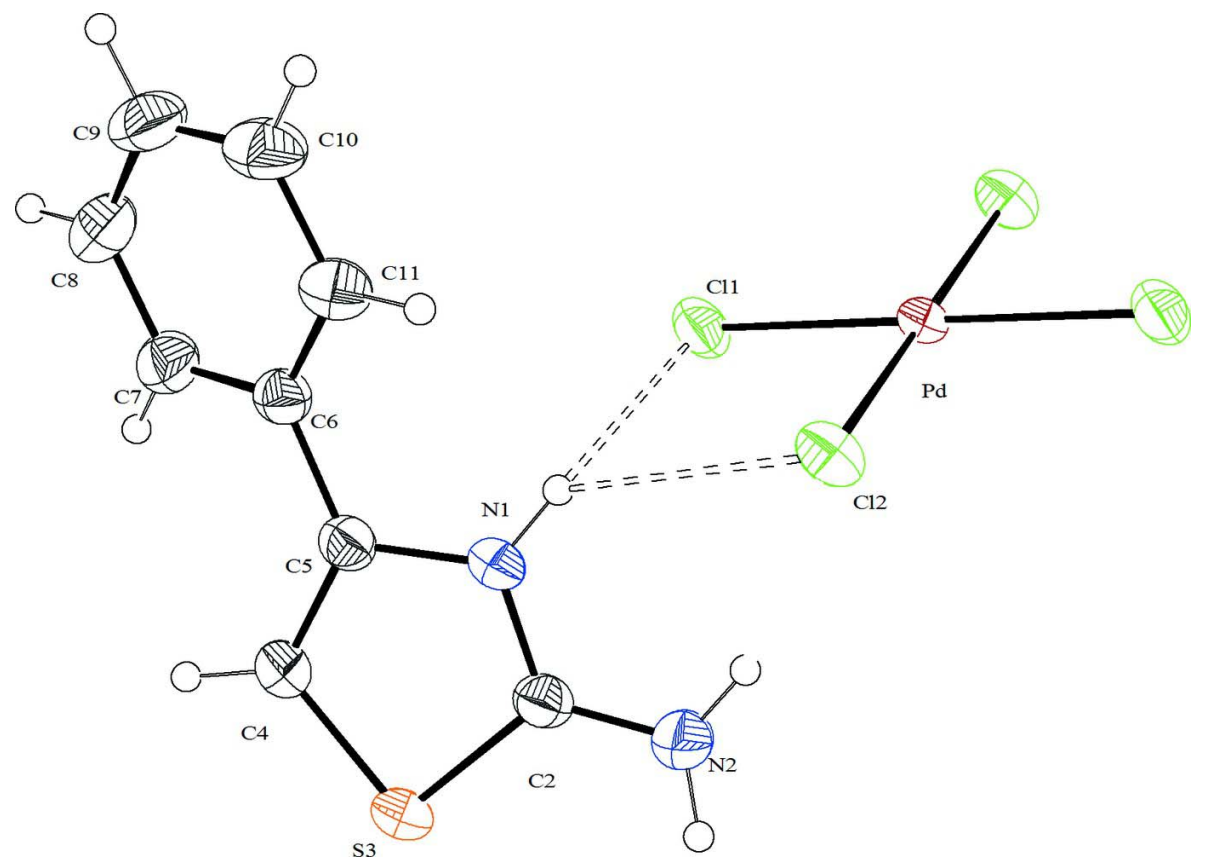

\section{Figure 1}

Molecular structure of title compound with displacement ellipsoids at the $40 \%$ probability. Hydrogen atoms are drawn as spheres of arbitrary radius.

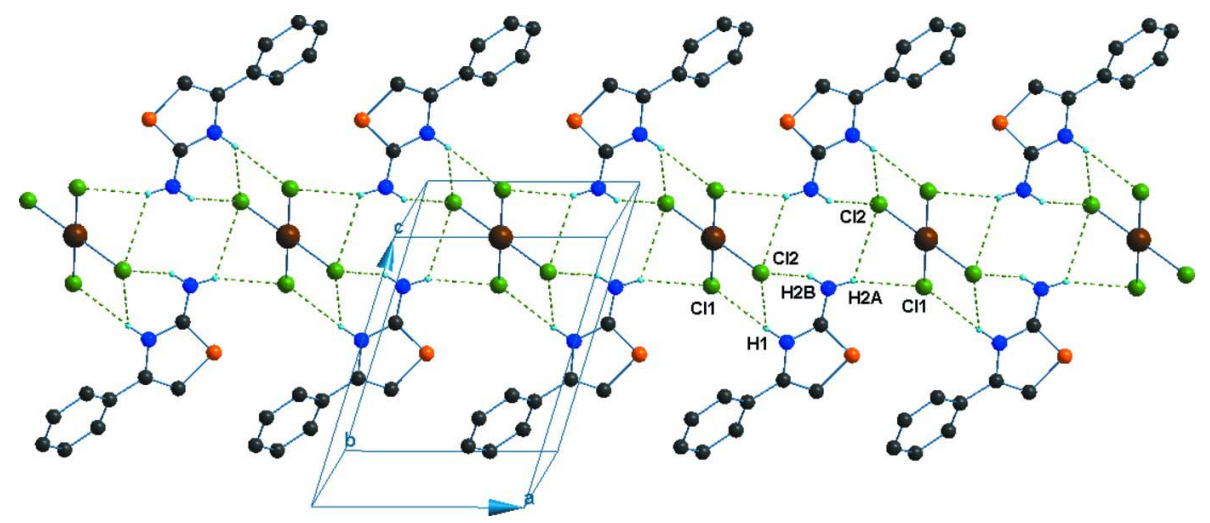

\section{Figure 2}

Linear arrangement due hydrogen bond patterns in crystal structure of the title compound.

\section{Bis(2-amino-4-phenyl-1,3-thiazol-3-ium) tetrachloridopalladate(II)}

Crystal data

$\left(\mathrm{C}_{9} \mathrm{H}_{9} \mathrm{~N}_{2} \mathrm{~S}\right)_{2}\left[\mathrm{PdCl}_{4}\right]$

Triclinic, $P \overline{1}$

$M_{r}=602.68$

$a=7.2880$ (2) $\AA$ 


$$
\begin{aligned}
& b=8.9214(3) \AA \\
& c=9.8192(3) \AA \\
& \alpha=66.258(1)^{\circ} \\
& \beta=73.778(1)^{\circ} \\
& \gamma=84.468(1)^{\circ} \\
& V=561.04(3) \AA^{3} \\
& Z=1 \\
& F(000)=300
\end{aligned}
$$

\section{Data collection}

Bruker APEXII CCD area-detector diffractometer

Detector resolution: 0.83 pixels $\mathrm{mm}^{-1}$ $\omega$ scans

Absorption correction: analytical (SADABS; Bruker, 2012)

$T_{\min }=0.658, T_{\max }=0.842$

4857 measured reflections

\section{Refinement}

Refinement on $F^{2}$

Least-squares matrix: full

$R\left[F^{2}>2 \sigma\left(F^{2}\right)\right]=0.020$

$w R\left(F^{2}\right)=0.051$

$S=1.11$

2060 reflections

143 parameters

3 restraints

Hydrogen site location: mixed
$D_{\mathrm{x}}=1.784 \mathrm{Mg} \mathrm{m}^{-3}$

Mo $K \alpha$ radiation, $\lambda=0.71073 \AA$

Cell parameters from 4506 reflections

$\theta=2.4-25.4^{\circ}$

$\mu=1.50 \mathrm{~mm}^{-1}$

$T=298 \mathrm{~K}$

Prism, brown

$0.46 \times 0.28 \times 0.21 \mathrm{~mm}$

2060 independent reflections

1982 reflections with $I>2 \sigma(I)$

$R_{\text {int }}=0.026$

$\theta_{\text {max }}=25.4^{\circ}, \theta_{\text {min }}=2.4^{\circ}$

$h=-8 \rightarrow 8$

$k=-10 \rightarrow 10$

$l=-11 \rightarrow 11$

$\mathrm{H}$ atoms treated by a mixture of independent and constrained refinement

$w=1 /\left[\sigma^{2}\left(F_{\mathrm{o}}{ }^{2}\right)+(0.027 P)^{2}+0.0969 P\right]$ where $P=\left(F_{\mathrm{o}}{ }^{2}+2 F_{\mathrm{c}}{ }^{2}\right) / 3$

$(\Delta / \sigma)_{\max }<0.001$

$\Delta \rho_{\max }=0.26 \mathrm{e} \AA^{-3}$

$\Delta \rho_{\min }=-0.30$ e $\AA^{-3}$

Extinction correction: SHELXL97 (Sheldrick, 2008), $\mathrm{Fc}^{*}=\mathrm{kFc}\left[1+0.001 \mathrm{xFc}^{2} \lambda^{3} / \sin (2 \theta)\right]^{-1 / 4}$

Extinction coefficient: 0.015 (2)

\section{Special details}

Geometry. All e.s.d.'s (except the e.s.d. in the dihedral angle between two 1.s. planes) are estimated using the full covariance matrix. The cell e.s.d.'s are taken into account individually in the estimation of e.s.d.'s in distances, angles and torsion angles; correlations between e.s.d.'s in cell parameters are only used when they are defined by crystal symmetry. An approximate (isotropic) treatment of cell e.s.d.'s is used for estimating e.s.d.'s involving 1.s. planes.

Fractional atomic coordinates and isotropic or equivalent isotropic displacement parameters $\left(\AA^{2}\right)$

\begin{tabular}{lllll}
\hline & $x$ & $y$ & $z$ & $U_{\text {iso }} * / U_{\text {eq }}$ \\
\hline Pd & 0.5000 & 0.0000 & 1.0000 & $0.03103(10)$ \\
Cl1 & $0.54163(7)$ & $0.23160(6)$ & $0.77534(6)$ & $0.04971(15)$ \\
C12 & $0.78000(7)$ & $-0.10267(6)$ & $0.89335(6)$ & $0.04577(14)$ \\
$\mathrm{N} 1$ & $0.9774(2)$ & $0.2276(2)$ & $0.56952(19)$ & $0.0382(4)$ \\
$\mathrm{H} 1$ & $0.8651(19)$ & $0.185(3)$ & $0.628(2)$ & $0.046^{*}$ \\
$\mathrm{C} 2$ & $1.1103(3)$ & $0.2483(2)$ & $0.6301(2)$ & $0.0380(4)$ \\
$\mathrm{N} 2$ & $1.0983(3)$ & $0.1821(2)$ & $0.7791(2)$ & $0.0527(5)$ \\
$\mathrm{H} 2 \mathrm{~A}$ & $1.203(2)$ & $0.195(3)$ & $0.804(3)$ & $0.063 *$ \\
$\mathrm{H} 2 \mathrm{~B}$ & $1.001(3)$ & $0.112(3)$ & $0.837(3)$ & $0.063^{*}$ \\
$\mathrm{~S} 3$ & $1.29130(7)$ & $0.37696(7)$ & $0.49056(6)$ & $0.04726(15)$ \\
$\mathrm{C} 4$ & $1.1739(3)$ & $0.4022(3)$ & $0.3520(2)$ & $0.0459(5)$ \\
$\mathrm{H} 4$ & 1.2196 & 0.4690 & 0.2481 & $0.055^{*}$ \\
$\mathrm{C} 5$ & $1.0096(3)$ & $0.3149(2)$ & $0.4107(2)$ & $0.0365(4)$
\end{tabular}




\begin{tabular}{lllll} 
C6 & $0.8732(3)$ & $0.3009(2)$ & $0.3309(2)$ & $0.0374(4)$ \\
C7 & $0.8497(3)$ & $0.4318(3)$ & $0.2005(2)$ & $0.0484(5)$ \\
H7 & 0.9175 & 0.5291 & 0.1660 & $0.058^{*}$ \\
C8 & $0.7259(4)$ & $0.4194(3)$ & $0.1206(3)$ & $0.0572(6)$ \\
H8 & 0.7096 & 0.5087 & 0.0340 & $0.069^{*}$ \\
C9 & $0.6278(4)$ & $0.2758(3)$ & $0.1694(3)$ & $0.0585(6)$ \\
H9 & 0.5474 & 0.2666 & 0.1143 & $0.070^{*}$ \\
C10 & $0.6482(3)$ & $0.1453(3)$ & $0.2995(3)$ & $0.0576(6)$ \\
H10 & 0.5800 & 0.0484 & 0.3328 & $0.069^{*}$ \\
C11 & $0.7696(3)$ & $0.1563(3)$ & $0.3820(3)$ & $0.0475(5)$ \\
H11 & 0.7815 & 0.0678 & 0.4707 & $0.057^{*}$ \\
\hline
\end{tabular}

Atomic displacement parameters $\left(\AA^{2}\right)$

\begin{tabular}{lllllll}
\hline & $U^{11}$ & $U^{22}$ & $U^{33}$ & $U^{12}$ & $U^{13}$ & $U^{23}$ \\
\hline Pd & $0.02640(13)$ & $0.03439(14)$ & $0.03019(13)$ & $-0.00791(8)$ & $-0.00582(8)$ & $-0.00981(9)$ \\
C11 & $0.0358(3)$ & $0.0484(3)$ & $0.0431(3)$ & $-0.0070(2)$ & $-0.0057(2)$ & $0.0025(2)$ \\
C12 & $0.0345(3)$ & $0.0472(3)$ & $0.0495(3)$ & $-0.0033(2)$ & $-0.0015(2)$ & $-0.0183(2)$ \\
N1 & $0.0298(8)$ & $0.0420(9)$ & $0.0380(9)$ & $-0.0078(7)$ & $-0.0058(7)$ & $-0.0112(7)$ \\
C2 & $0.0326(9)$ & $0.0374(10)$ & $0.0416(11)$ & $-0.0027(8)$ & $-0.0092(8)$ & $-0.0129(8)$ \\
N2 & $0.0494(11)$ & $0.0594(12)$ & $0.0434(10)$ & $-0.0154(9)$ & $-0.0169(9)$ & $-0.0074(9)$ \\
S3 & $0.0326(3)$ & $0.0601(3)$ & $0.0447(3)$ & $-0.0143(2)$ & $-0.0069(2)$ & $-0.0151(2)$ \\
C4 & $0.0387(11)$ & $0.0584(13)$ & $0.0365(10)$ & $-0.0118(9)$ & $-0.0051(9)$ & $-0.0147(9)$ \\
C5 & $0.0321(9)$ & $0.0394(10)$ & $0.0376(10)$ & $-0.0008(8)$ & $-0.0062(8)$ & $-0.0164(8)$ \\
C6 & $0.0329(9)$ & $0.0436(10)$ & $0.0393(10)$ & $0.0001(8)$ & $-0.0064(8)$ & $-0.0217(9)$ \\
C7 & $0.0532(13)$ & $0.0504(12)$ & $0.0449(12)$ & $-0.0054(10)$ & $-0.0144(10)$ & $-0.0197(10)$ \\
C8 & $0.0645(15)$ & $0.0669(15)$ & $0.0498(13)$ & $0.0047(12)$ & $-0.0254(12)$ & $-0.0265(12)$ \\
C9 & $0.0519(14)$ & $0.0780(17)$ & $0.0680(16)$ & $0.0034(12)$ & $-0.0260(12)$ & $-0.0449(14)$ \\
C10 & $0.0501(13)$ & $0.0593(14)$ & $0.0773(17)$ & $-0.0071(11)$ & $-0.0169(12)$ & $-0.0389(13)$ \\
C11 & $0.0427(11)$ & $0.0453(11)$ & $0.0573(13)$ & $-0.0019(9)$ & $-0.0134(10)$ & $-0.0223(10)$ \\
& & & & & & \\
\hline
\end{tabular}

Geometric parameters $\left(\AA,{ }^{o}\right)$

\begin{tabular}{llll}
\hline $\mathrm{Pd}-\mathrm{Cl1}$ & $2.3031(5)$ & $\mathrm{C} 4-\mathrm{H} 4$ & 0.9300 \\
$\mathrm{Pd}-\mathrm{Cl1} 1^{\mathrm{i}}$ & $2.3031(5)$ & $\mathrm{C} 5-\mathrm{C} 6$ & $1.468(3)$ \\
$\mathrm{Pd}-\mathrm{Cl} 2^{\mathrm{i}}$ & $2.3061(5)$ & $\mathrm{C} 6-\mathrm{C} 7$ & $1.383(3)$ \\
$\mathrm{Pd}-\mathrm{Cl} 2$ & $2.3061(5)$ & $\mathrm{C} 6-\mathrm{C} 11$ & $1.393(3)$ \\
$\mathrm{N} 1-\mathrm{C} 2$ & $1.331(2)$ & $\mathrm{C} 7-\mathrm{C} 8$ & $1.388(3)$ \\
$\mathrm{N} 1-\mathrm{C} 5$ & $1.395(3)$ & $\mathrm{C} 7-\mathrm{H} 7$ & 0.9300 \\
$\mathrm{~N} 1-\mathrm{H} 1$ & $0.876(10)$ & $\mathrm{C} 8-\mathrm{C} 9$ & $1.369(4)$ \\
$\mathrm{C} 2-\mathrm{N} 2$ & $1.319(3)$ & $\mathrm{C} 8-\mathrm{H} 8$ & 0.9300 \\
$\mathrm{C} 2-\mathrm{S} 3$ & $1.7179(19)$ & $\mathrm{C} 9-\mathrm{C} 10$ & $1.373(4)$ \\
$\mathrm{N} 2-\mathrm{H} 2 \mathrm{~A}$ & $0.894(10)$ & $\mathrm{C} 9-\mathrm{H} 9$ & 0.9300 \\
$\mathrm{~N} 2-\mathrm{H} 2 \mathrm{~B}$ & $0.887(10)$ & $\mathrm{C} 10-\mathrm{C} 11$ & $1.389(3)$ \\
$\mathrm{S} 3-\mathrm{C} 4$ & $1.733(2)$ & $\mathrm{C} 10-\mathrm{H} 10$ & 0.9300 \\
$\mathrm{C} 4-\mathrm{C} 5$ & $1.343(3)$ & $\mathrm{C} 11-\mathrm{H} 11$ & 0.9300 \\
& & & $129.09(18)$ \\
$\mathrm{C} 11-\mathrm{Pd}-\mathrm{C} 11^{\mathrm{i}}$ & 180.0 & $\mathrm{C} 4-\mathrm{C} 5-\mathrm{C} 6$ &
\end{tabular}




$\begin{array}{llll}\mathrm{C} 11-\mathrm{Pd}-\mathrm{Cl2} & \mathrm{N} 1-\mathrm{C} 5-\mathrm{C} 6 & 120.10(17) \\ \mathrm{C} 11^{\mathrm{i}}-\mathrm{Pd}-\mathrm{Cl} 2^{\mathrm{i}} & 90.134(19) & \mathrm{C} 7-\mathrm{C} 6-\mathrm{C} 11 & 119.06(19) \\ \mathrm{C} 11-\mathrm{Pd}-\mathrm{Cl} 2 & 89.866(19) & \mathrm{C} 7-\mathrm{C} 6-\mathrm{C} 5 & 119.70(18) \\ \mathrm{C} 11^{\mathrm{i}}-\mathrm{Pd}-\mathrm{Cl} 2 & 89.866(19) & \mathrm{C} 11-\mathrm{C} 6-\mathrm{C} 5 & 121.22(19) \\ \mathrm{C} 12^{\mathrm{i}}-\mathrm{Pd}-\mathrm{Cl} 2 & 90.134(19) & \mathrm{C} 6-\mathrm{C} 7-\mathrm{C} 8 & 120.7(2) \\ \mathrm{C} 2-\mathrm{N} 1-\mathrm{C} 5 & 180.00(2) & \mathrm{C} 6-\mathrm{C} 7-\mathrm{H} 7 & 119.7 \\ \mathrm{C} 2-\mathrm{N} 1-\mathrm{H} 1 & 115.25(16) & \mathrm{C} 8-\mathrm{C} 7-\mathrm{H} 7 & 119.7 \\ \mathrm{C} 5-\mathrm{N} 1-\mathrm{H} 1 & 120.8(15) & \mathrm{C} 9-\mathrm{C} 8-\mathrm{C} 7 & 120.0(2) \\ \mathrm{N} 2-\mathrm{C} 2-\mathrm{N} 1 & 121.9(15) & \mathrm{C} 9-\mathrm{C} 8-\mathrm{H} 8 & 120.0 \\ \mathrm{~N} 2-\mathrm{C} 2-\mathrm{S} 3 & 123.49(18) & \mathrm{C} 7-\mathrm{C} 8-\mathrm{H} 8 & 120.0(2) \\ \mathrm{N} 1-\mathrm{C} 2-\mathrm{S} 3 & 125.28(16) & \mathrm{C} 8-\mathrm{C} 9-\mathrm{C} 10 & 120.0 \\ \mathrm{C} 2-\mathrm{N} 2-\mathrm{H} 2 \mathrm{~A} & 111.19(14) & \mathrm{C} 10-\mathrm{C} 9-\mathrm{H} 9 & 120.0 \\ \mathrm{C} 2-\mathrm{N} 2-\mathrm{H} 2 \mathrm{~B} & 114.7(17) & \mathrm{C} 9-\mathrm{C} 10-\mathrm{C} 11 & 119.6 \\ \mathrm{H} 2 \mathrm{~A}-\mathrm{N} 2-\mathrm{H} 2 \mathrm{~B} & 115.3(18) & \mathrm{C} 9-\mathrm{C} 10-\mathrm{H} 10 & 119.6 \\ \mathrm{C} 2-\mathrm{S} 3-\mathrm{C} 4 & 128(2) & \mathrm{C} 11-\mathrm{C} 10-\mathrm{H} 10 & 119.5(2) \\ \mathrm{C} 5-\mathrm{C} 4-\mathrm{S} 3 & 90.03(10) & \mathrm{C} 10-\mathrm{C} 11-\mathrm{C} 6 & 120.2 \\ \mathrm{C} 5-\mathrm{C} 4-\mathrm{H} 4 & 112.71(16) & \mathrm{C} 10-\mathrm{C} 11-\mathrm{H} 11 & 120.2 \\ \mathrm{~S} 3-\mathrm{C} 4-\mathrm{H} 4 & 123.6 & \mathrm{C} 6-\mathrm{C} 11-\mathrm{H} 11 & \\ \mathrm{C} 4-\mathrm{C} 5-\mathrm{N} 1 & 123.6 & & \end{array}$

Symmetry code: (i) $-x+1,-y,-z+2$.

Hydrogen-bond geometry $\left(\AA,{ }^{\circ}\right)$

\begin{tabular}{lllll}
\hline$D-\mathrm{H} \cdots A$ & $D-\mathrm{H}$ & $\mathrm{H} \cdots A$ & $D \cdots A$ & $D-\mathrm{H} \cdots A$ \\
\hline $\mathrm{N} 2-\mathrm{H} 2 B \cdots \mathrm{Cl} 2$ & $0.89(1)$ & $2.41(2)$ & $3.237(2)$ & $155(2)$ \\
$\mathrm{N} 2-\mathrm{H} 2 A \cdots \mathrm{Cl} 2^{\mathrm{ii}}$ & $0.89(1)$ & $2.78(2)$ & $3.3572(19)$ & $123(2)$ \\
$\mathrm{N} 2-\mathrm{H} 2 A \cdots \mathrm{Cl} 1^{\mathrm{iii}}$ & $0.89(1)$ & $2.44(1)$ & $3.291(2)$ & $159(2)$ \\
$\mathrm{N} 1-\mathrm{H} 1 \cdots \mathrm{Cl} 2$ & $0.88(1)$ & $2.79(2)$ & $3.4028(17)$ & $129(2)$ \\
$\mathrm{N} 1-\mathrm{H} 1 \cdots \mathrm{C} 11$ & $0.88(1)$ & $2.49(2)$ & $3.2593(17)$ & $147(2)$ \\
\hline
\end{tabular}

Symmetry codes: (ii) $-x+2,-y,-z+2$; (iii) $x+1, y, z$. 